\title{
Generalized Variability Orderings among Nonnegative Fuzzy Random Variables
}

\author{
S. Ramasubramanian and P. Mahendran \\ Department of Mathematics, Anna University, BIT Campus, Tiruchirappalli 620024, India \\ Correspondence should be addressed to P. Mahendran; p.mahendran@mail.aubit.edu.in
}

Received 9 October 2015; Revised 24 November 2015; Accepted 7 December 2015

Academic Editor: Naseer Shahzad

Copyright (C) 2015 S. Ramasubramanian and P. Mahendran. This is an open access article distributed under the Creative Commons Attribution License, which permits unrestricted use, distribution, and reproduction in any medium, provided the original work is properly cited.

\begin{abstract}
The variability ordering for more and less variables of fuzzy random variables in terms of its distribution function is defined. A property of new better than used in expectation (NBUE) and new worse than used in expectation (NWUE) is derived as an application to the variability ordering of fuzzy random variables. The concept of generalized variability orderings of nonnegative fuzzy random variables representing lifetime of components is introduced. The $<^{P}$ domination is a generalized variability ordering. We proposed an integral inequality to the case of fuzzy random variables using $<^{P}$ ordering. The results included equivalent conditions which justify the generalized variability orderings.
\end{abstract}

\section{Introduction}

Fuzzy random variables generalize random variables and random sets. Kwakernaak $[1,2]$ introduced the concept of a fuzzy random variable as a function $X: \Omega \rightarrow F(R)$ where $(\Omega, A, P)$ is a probability space and $F(R)$ denote the set of all canonical fuzzy numbers. Puri and Ralescu [3] defined the notion of fuzzy random variable as a function $X: \Omega \rightarrow F\left(R^{n}\right)$ where $(\Omega, A, P)$ is a probability space and $F\left(R^{n}\right)$ denotes all functions $U: R^{n} \rightarrow[0,1]$. The lifetimes of machines and components are expressed in terms of linguistic variables with imprecise boundaries. In such cases, fuzzy random variables are only capable of dealing with the vague data. Life distributions represented by nonnegative random variables and their distributions can be ordered by the comparison of their properties. Assessing the credibility of components in terms of their lifetime through fuzzy random variables is more realistic and appropriate because consumers express their opinion only in terms of linguistic variables with regard to the lifetimes of appliances and components. Bhattacharjee [4] investigated a generalized variability ordering and its weaker versions among nonnegative random variables representing lifetime of components. Abouammoh [5] studied on the criteria of the mean residual lifetime. Bhattacharjee and Sethuraman [6] have considered the families of life distributions characterized by two moments. Piriyakumar and Renganathan [7] employed fuzzy random variables to define various stochastic orderings. Ramasubramanian and Mahendran [8] studied the hazard rate, mean residual life ordering, and bivariate characterization of hazard rate ordering of fuzzy random variables.

In this paper the variability ordering of two fuzzy random variables $X$ and $Y$ in the sense of more and less variables of fuzzy random variables in terms of its distribution function is defined. The application to the variability ordering of fuzzy random variables, a property of new better than used in expectation (NBUE) and new worse than used in expectation (NWUE), is achieved. The concept of generalized variability orderings of nonnegative fuzzy random variables is commenced. The purpose of this paper is to investigate the implications of $<^{P}$ ordered lifetimes represented by fuzzy random variables. The $<^{P}$ domination is a generalized variability ordering. A useful integral inequality to the case of fuzzy random variables using $<^{P}$ ordering is suggested. The results consisting of the equivalent conditions defend the generalized variability ordering. These orderings are employed to compare the appealing natures of different risks and are essential in the theory of decision making under uncertainty. 
The fuzzy random variables $X$ and $Y$ are considered as the lifetime of components. The variability ordering and generalized variability ordering are useful to compare the fuzzy random variables, also in terms of their respective lifetime distributions. Variability orderings are used to compare probability distributions according to their spread or dispersion. If two variables have the same mean, they can still be compared by spreading out of their distributions. This is captured to a limited extent by the variance, but more fully by a range of stochastic orders. For generalized variability orderings, $<^{P}$ ordered lifetimes of fuzzy random variables which must have a pth moment. Ross and Schechner [9] proved the property of the variability ordering and applied it to branching processes and shock models and also to a population survival and to Poisson shock model. Life distributions and their variations have been introduced in reliability theory; the applications of these classes of life distributions have been seen in engineering, social, biological science, and so forth. Reliability analysis has shown a growing interest in modeling of survival data using classifications of life distributions based on some aspects of ageing [10].

For the families of life distributions, Barlow and Proschan [10] have proved the following chain of implication: IFR $\Rightarrow$ IFRA $\Rightarrow \mathrm{NBU} \Rightarrow$ NBUE and DFR $\Rightarrow$ DFRA $\Rightarrow$ NWU $\Rightarrow$ NWUE. In this chain of implication, new better than used in expectation (NBUE) is the extended version of increasing failure rate (IFR), increasing failure rate average (IFRA), and new better than used (NBU). Similarly, new worse than used in expectation (NWUE) is also the extended version of the decreasing failure rate (DFR), decreasing failure rate average (DFRA), and new worse than used (NWU).

NBUE (NWUE) is used to compare the ageing properties of two arbitrary life distributions. Lorenzo et al. [11] have used NBUE property for estimation of distributions. The simple ageing concepts such as IFR, IFRA, NBU, and NBUE are proved useful in reliability related decision making, such as in replacement and maintenance studies. In this paper, a property of new better than used in expectation (NBUE) and new worse than used in expectation (NWUE) is derived as an application to the variability ordering of fuzzy random variables (Theorem 11).

This paper is organized in the following manner. In Section 2, the preliminaries of fuzzy umbers and fuzzy random variables are explained. In Section 3, the concept of variability ordering and its related definitions are discussed and a property of NBUE and NWUE is obtained as an application to the variability ordering of fuzzy random variables. In Section 4, the concept of generalized variability ordering is introduced to the case of Kwakernaak's fuzzy random variables and the derived equivalent conditions that justify the generalized variability orderings.

\section{Preliminaries}

Definition 1. Let $X$ be a universal set; then a fuzzy set $\widetilde{A}=$ $\left\{\left(x, \mu_{A}(x)\right) / x \in X\right\}$ of $X$ is defined by its membership function $\mu_{\widetilde{A}}: X \rightarrow[0,1]$; see $[12]$.
Definition 2. For each $0 \leq \alpha \leq 1$, the $\alpha$-cut set of $\widetilde{A}$ is denoted by $\widetilde{A}_{\alpha}=\left\{x \in X: \mu_{\widetilde{A}}(x) \geq \alpha\right\}[12]$.

Definition 3. A fuzzy number is a fuzzy set of $R$ such that the following conditions are satisfied:

(i) $\widetilde{A}$ is normal if there exist $x \in X$ such that $\mu_{\widetilde{A}}(x)=1$.

(ii) $\widetilde{A}$ is called convex if $\mu_{\widetilde{A}}\left(\lambda x_{1}+\left(1-\lambda x_{2}\right)\right) \geq$ $\min \left(\mu_{\widetilde{A}}\left(x_{1}\right), \mu_{\widetilde{A}}\left(x_{2}\right)\right)$.

(iii) $\widetilde{A}$ is called upper semicontinuous with compact support; that is, for every $\epsilon>0$ there exist $\delta>0$; $|x-y|<\delta \Rightarrow \mu_{\widetilde{A}}(x)<\mu_{\widetilde{A}}(y)+\epsilon$.

(iv) The $\alpha$-cut of a fuzzy number is a closed interval denoted by $A_{\alpha}=\left[A_{\alpha}^{L}, A_{\alpha}^{U}\right]$ where $A_{\alpha}^{L}=\inf \{x \in$ $\left.R ; \mu_{\widetilde{A}}(x) \geq \alpha\right\}$ and $A_{\alpha}^{U}=\sup \left\{x \in R ; \mu_{\widetilde{A}}(x) \geq \alpha\right\}$.

(v) If $\widetilde{A}$ is a closed and bounded fuzzy number with $A_{\alpha}^{L}$, $A_{\alpha}^{U}$ and its membership function is strictly increasing on $\left[A_{\alpha}^{L}, A_{1}^{L}\right]$ and strictly decreasing on $\left[A_{1}^{U}, A_{\alpha}^{U}\right]$ then $\widetilde{A}$ is called canonical fuzzy number.

Definition 4. Let $(\Omega, f, P)$ be a probability space. A fuzzy random variable $X: \Omega \rightarrow F(R)$, where $F(R)$ is the set of fuzzy numbers in $R$ (i.e., for $w \in \Omega, X(w) \in F(R)$ ) that satisfies the following properties. For $\alpha \in(0,1]$ and $w \in \Omega$ both $X_{\alpha}^{\star}$ and $X_{\alpha}^{\star *}$ defined as $X_{\alpha}^{\star}(w)=\inf X_{\alpha}(w)$ and $X_{\alpha}^{* \star}(w)=$ Sup $X_{\alpha}(w)$ are finite real valued random variables on $(\Omega, f, P)$ such that the mathematical expectations $E X_{\alpha}^{\star}$ and $E X_{\alpha}^{\star *}$ exist. For $\alpha \in(0,1], X_{\alpha}^{\star} \in X_{\alpha}(w)$ and $X_{\alpha}^{\star *} \in X_{\alpha}(w)[1,2]$.

If $u_{o}$ is the latent variable and $w \in \Omega$ is the outcome of the experiment, the exact value of $u_{o}(w)$ is unobservable and it is only known through a fuzzy number with which $u_{o}(w)$ is submerged in Kwakernaak $[1,2]$ construction of fuzzy random variables; random variable found in the basic set is required to exhibit a type of $\sigma(x)$-measurability. Due to this kind of construction many $f$ measurable random variables may be abandoned. Nevertheless we require some degree of structural compatibility between fuzzy perception and the underlying measure space. To accomplish this requirement Boswell and Taylor [13] have imposed an assumption that $\sigma(x)$ must constitute a subsigma algebra of $f$. If $U$ is a $f$-measurable random variable then $\mu_{X}(u)=\inf _{w \in \Omega} X_{w}(u(w))$ is the valuation of its suitability as a reconstruction of $u_{o}$. Let $U_{f}$ be the collection of all $f$-measurable random variables on $\Omega$. Then the fuzzy random variable induced by $X$ is defined as $X=$ $\left(U_{f}, \mu_{x}\right)$. The expectation and probability for the fuzzy random variable are defined as

$$
\begin{gathered}
\underset{E X}{\mu}(x)=\sup _{U \in U_{f}: E U=X} \inf _{w \in \Omega} X_{w}(U(w)), \\
\underset{P(x \in A)}{\mu}(p)=\sup _{U \in U_{f}: P(U \in A)=p} \inf _{w \in \Omega} X_{w}(U(w)) .
\end{gathered}
$$




\section{Variability Orderings for Fuzzy Random Variables}

Definition 5. The fuzzy random variables $X$ is said to be more variable (less variable) than the fuzzy random variable $Y$ if for $\alpha \in(0,1]$

$$
E\left[h\left(X_{\alpha}^{\star} \vee X_{\alpha}^{\star *}\right)\right] \geq(\leq) E\left[h\left(Y_{\alpha}^{\star} \vee Y_{\alpha}^{\star *}\right)\right]
$$

for all increasing convex function $h$. Symbolically one denotes it as $X \geq_{v} Y\left(X \leq_{v} Y\right)[14]$

Theorem 6. If $X$ and $Y$ are nonnegative fuzzy random variables with distribution functions $F$ and $G$, respectively, then $X \geq_{v} Y\left(X \leq_{v} Y\right)$ if and only if

$$
\int_{a}^{\infty} \bar{F}(x) d x \geq(\leq) \int_{a}^{\infty} \bar{G}(x) d x \quad \forall a \geq 0
$$

(see [14]).

Definition 7. $R(x / t)=\operatorname{Pr}(T>x+t / T>t)=\operatorname{Pr}(T>$ $x+t) / \operatorname{Pr}(T>t)=R(X+t) / R(t)$ is called the conditional survivor function of the item at age $t$. The mean residual life of the item at age $t$ is $\operatorname{MRL}(t)=\mu(t)=\int_{0}^{\infty} R(x / t) d x=(1 /$ $R(t)) \int_{t}^{\infty} R(x) d x[15]$.

Definition 8. A life distribution $F(x)$ is said to be new better than used in expectation ( $F$ is NBUE) if (i) $F$ has finite mean $\mu$ (ii) $\operatorname{MRL}(X) \leq \mu$. A life distribution $F(x)$ is said to be new worse than used in expectation ( $F$ is NWUE) if (i) $F$ has finite mean $\mu$ (ii) $\operatorname{MTTF}(X) \geq \mu[15]$.

Definition 9. Let $T$ denote the time to failure of an item, with the probability density $f(t)$ and survival function $R(t)$. The mean time to failure is the mean value of $T$ which is given by

$$
\operatorname{MTTF}=\int_{0}^{\infty} t \cdot f(t) d t=\int_{0}^{t} R(t) d t
$$

(see [15]).

Definition 10. The nonnegative fuzzy random variable $X$ is said to be new better than used in expectation (NBUE) if for $\alpha \in(0,1]$

$$
E\left[\frac{\left(X_{\alpha}^{\star}-a\right) \vee\left(X_{\alpha}^{\star *}-a\right)}{\left(X_{\alpha}^{\star} \vee X_{\alpha}^{\star *}\right)}>a\right] \leq E\left[X_{\alpha}^{\star} \vee X_{\alpha}^{\star *}\right]
$$

$$
\forall a \geq 0 \text {. }
$$

It is said to be new worse than used in expectation (NWUE) if for $\alpha \in(0,1]$

$$
E\left[\frac{\left(X_{\alpha}^{\star}-a\right) \vee\left(X_{\alpha}^{\star *}-a\right)}{\left(X_{\alpha}^{\star} \vee X_{\alpha}^{\star *}\right)}>a\right] \geq E[X] \quad \forall a \geq 0 .
$$

If $X$ is considered as the lifetime of some unit, then $X$ being NBUE (NWUE) means that the expected residual life of any used item is less (greater) than or equal to the expected life of a new item. If $X$ is NBUE and $F$ is the distribution of $X$, then we say that $F$ is an NBUE distribution and similar to NWUE.
Theorem 11. If $F$ is an (i) NWUE distribution with mean $\mu$, then $F \geq_{v} \exp (\mu)$, and if it is (ii) NBUE distribution with mean $\mu$ then $F \leq_{v} \exp (\mu)$ where $\exp (\mu)$ is the exponential distribution with mean $\mu$.

Proof of (i). Suppose $F$ is NWUE with mean $\mu$; we need to prove that

$$
\int_{c}^{\infty} \bar{F}(x) d x \geq \int_{c}^{\infty} e^{-x / \mu} d x \quad \forall c \geq 0 .
$$

If $X$ has distribution $F$, then for

$$
\begin{aligned}
E[ & \left.\frac{\left(X_{\alpha}^{\star}-a\right) \vee\left(X_{\alpha}^{\star *}-a\right)}{\left(X_{\alpha}^{\star} \vee X_{\alpha}^{\star *}\right)}>a\right] \\
& =\int_{0}^{\infty} P\left[\frac{\left(X_{\alpha}^{\star}-a\right) \vee\left(X_{\alpha}^{\star *}-a\right)>x}{\left(X_{\alpha}^{\star} \vee X_{\alpha}^{\star *}\right)>a}\right] d x \\
& =\int_{0}^{\infty} P\left[\frac{\left(X_{\alpha}^{\star} \vee X_{\alpha}^{\star *}\right)>a+x}{\left(X_{\alpha}^{\star} \vee X_{\alpha}^{\star *}\right)>a}\right] d x \\
& =\int_{0}^{\infty} \frac{\bar{F}(a+x)}{\bar{F}(a)} d x=\int_{a}^{\infty} \frac{\bar{F}(y)}{\bar{F}(a)} d y .
\end{aligned}
$$

$F$ is NWUE with mean $\mu$; we have $E\left[\left(X_{\alpha}^{\star}-a\right) \vee\left(X_{\alpha}^{\star *}-a\right) /\right.$ $\left.\left(X_{\alpha}^{\star} \vee X_{\alpha}^{\star *}\right)>a\right] \geq E[X]$.

Equation (8) implies

$$
\begin{aligned}
\int_{a}^{\infty} \frac{\bar{F}(y)}{\bar{F}(a)} d y & \geq E(X) \\
& \Longrightarrow \int_{a}^{\infty} \frac{\bar{F}(y)}{\bar{F}(a)} d y \geq \mu \\
& \Longrightarrow \frac{\bar{F}(a)}{\int_{a}^{\infty} \bar{F}(y) d y} \leq \frac{1}{\mu} \\
& \Longrightarrow \int_{0}^{c} \frac{\bar{F}(a)}{\int_{a}^{\infty} \bar{F}(y) d y} d a \leq \frac{1}{\mu} \int_{0}^{c} d a=\frac{c}{\mu} \\
& \Longrightarrow \int_{0}^{c} \frac{\bar{F}(a)}{\int_{a}^{\infty} \bar{F}(y) d y} d a \leq \frac{c}{\mu} .
\end{aligned}
$$

We can evaluate the left-hand side by making the change of variables

$$
\begin{aligned}
x & =\int_{a}^{\infty} \bar{F}(y) d y=\int_{a}^{\infty} d(\bar{F}(y))=\bar{F}(\infty)-\bar{F}(a), \\
x & =\bar{F}(a) \\
d x & =\bar{F}(a) d a .
\end{aligned}
$$

Taking $a=0$,

$$
x=\int_{0}^{\infty} \bar{F}(y) d y=E(X)=\mu .
$$


Taking $a=c$, we have

$$
x=\int_{c}^{\infty} \bar{F}(y) d y .
$$

We denote

$$
x(c)=\int_{c}^{\infty} \bar{F}(y) d y .
$$

Then (9) takes up the form

$$
\begin{gathered}
-\int_{\mu}^{x(c)} \frac{d x}{x} \leq \frac{c}{\mu} \Longrightarrow-\left.\log x\right|_{\mu} ^{x(c)} \leq \frac{c}{\mu} \\
\Longrightarrow-\log \left[\frac{\int_{\mathcal{c}}^{\infty} \bar{F}(y) d y}{\mu}\right] \leq \frac{c}{\mu} \\
\Longrightarrow \log \left[\frac{\int_{\mathcal{c}}^{\infty} \bar{F}(y) d y}{\mu}\right] \geq-\frac{c}{\mu} \\
\Longrightarrow \frac{\int_{\mathcal{c}}^{\infty} \bar{F}(y) d y}{\mu} \geq e^{-x / \mu} \\
\Longrightarrow \int_{c}^{\infty} \bar{F}(y) d y \geq \mu e^{-x / \mu} \\
\Longrightarrow \int_{c}^{\infty} \bar{F}(y) d y \geq \int_{\mathcal{c}}^{\infty} e^{-x / \mu} d x
\end{gathered}
$$

$$
c \geq 0 .
$$

After proving (ii) NBUE distribution with mean $\mu$ then $F \leq_{v} \exp (\mu)$ is similar to the above.

\section{Generalized Variability Ordering for Fuzzy Random Variables}

The investigation of generalized variability ordering and its weaker versions among nonnegative fuzzy random variables representing lifetime components are stated in this section.

Definition 12. Let $X, Y$ be nonnegative fuzzy random variables representing lifetimes with distribution functions $F$ and $G$, respectively. One says $F<^{P} G$ with a finite moment of order $p>0$, if the survival functions satisfy

$$
\begin{aligned}
& \int_{t}^{\infty} X^{P-1}\left[P\left\{\left(Y_{\alpha}^{*}-X_{\alpha}^{*}\right) \vee\left(Y_{\alpha}^{* *}-X_{\alpha}^{* *}\right)\right\}>x\right] d x \\
& \quad \geq 0 .
\end{aligned}
$$

For $t>0$ and for each $\alpha \in(0,1]$, we say that the fuzzy random variables $X<^{P} Y \Leftrightarrow F<^{P} G$.

Consider the following relation $<^{P}$ among life distributions defined by using condition (15).
Definition 13. $F<^{(P)} G$ if

$$
\begin{aligned}
\int_{t}^{\infty} & X^{P-1}\left[P\left\{\left(X_{\alpha}^{*}-x\right) \vee\left(X_{\alpha}^{* *}-x\right)\right\}>0\right] d x \\
\leq & \int_{t}^{\infty} X^{P-1}\left[P\left\{\left(Y_{\alpha}^{*}-x\right) \vee\left(Y_{\alpha}^{* *}-x\right)\right\}>0\right] d x \\
& <\infty
\end{aligned}
$$

for $t \geq 0$ and for each $\alpha \in(0,1]$.

Theorem 14. If $X$ and $Y$ are nonnegative fuzzy random variables with distribution functions $F$ and $G$, respectively, then $X \geq_{v} Y$ if and only if

$$
\begin{aligned}
& \int_{a}^{\infty}\left[P\left\{\left(X_{\alpha}^{*}-x\right) \vee\left(X_{\alpha}^{* *}-x\right)\right\}>0\right] d x \\
& \quad \geq \int_{a}^{\infty}\left[P\left\{\left(Y_{\alpha}^{*}-x\right) \vee\left(Y_{\alpha}^{* *}-x\right)\right\}>0\right] d x
\end{aligned}
$$

for all $a \geq 0$ and $\alpha \in(0,1]$.

Proof. We assume that $X \geq_{v} Y$.

Define the function $h_{a}$ as

$$
h_{a}(x)=(x-a)^{+}= \begin{cases}0 ; & x \leq a \\ x-a ; & x>a .\end{cases}
$$

$h_{a}$ is the convex increasing function:

$$
\begin{aligned}
& \therefore E\left[h_{a}(X)\right] \geq E\left[h_{a}(Y)\right] \\
& \begin{aligned}
E & {\left[h_{a}(X)\right]=\int_{0}^{\infty} P\left\{\left[\left(X_{\alpha}^{*}-a\right) \vee\left(X_{\alpha}^{* *}-a\right)\right]>x\right\} d x } \\
& =\int_{0}^{\infty} P\left\{\left[X_{\alpha}^{*} \vee X_{\alpha}^{* *}\right]>a+x\right\} d x \\
& =\int_{a}^{\infty} P\left\{\left[X_{\alpha}^{*} \vee X_{\alpha}^{* *}\right]>y\right\} d y \\
& =\int_{a}^{\infty} P\left\{\left[\left(X_{\alpha}^{*}-x\right) \vee\left(X_{\alpha}^{* *}-x\right)\right]>0\right\} d x .
\end{aligned}
\end{aligned}
$$

Similarly

$$
E\left[h_{a}(Y)\right]=\int_{a}^{\infty} P\left\{\left[\left(Y_{\alpha}^{*}-x\right) \vee\left(Y_{\alpha}^{* *}-x\right)\right]>0\right\} d x .
$$

Conversely assume that

$$
\begin{aligned}
& \int_{a}^{\infty} P\left\{\left[\left(X_{\alpha}^{*}-x\right) \vee\left(X_{\alpha}^{* *}-x\right)\right]>0\right\} d x \\
& \quad \geq \int_{a}^{\infty} P\left\{\left[\left(Y_{\alpha}^{*}-x\right) \vee\left(Y_{\alpha}^{* *}-x\right)\right]>0\right\} d x
\end{aligned}
$$

for all $a \geq 0$ and $\alpha \in(0,1]$. 
Let $h$ be a convex function, and suppose that $h$ is twice differentiable. Since $h$ is convex and $h^{\prime \prime} \geq 0$,

$$
\begin{gathered}
\int_{0}^{\infty} h^{\prime \prime}(a) \int_{a}^{\infty} P\left\{\left[\left(X_{\alpha}^{*}-x\right) \vee\left(X_{\alpha}^{* *}-x\right)\right]>0\right\} d x d a \\
\geq \int_{0}^{\infty} h^{\prime \prime}(a) \\
\cdot \int_{a}^{\infty} P\left\{\left[\left(Y_{\alpha}^{*}-x\right) \vee\left(Y_{\alpha}^{* *}-x\right)\right]>0\right\} d x d a .
\end{gathered}
$$

\section{Consider}

$$
\begin{aligned}
& \int_{0}^{\infty} h^{\prime \prime}(a) \int_{a}^{\infty} P\left\{\left[\left(X_{\alpha}^{*}-x\right) \vee\left(X_{\alpha}^{* *}-x\right)\right]>0\right\} d x d a \\
& =\int_{0}^{\infty} \int_{a}^{x}\left(h^{\prime \prime}(a) d a\right) P\left\{\left[\left(X_{\alpha}^{*}-x\right) \vee\left(X_{\alpha}^{* *}-x\right)\right]\right. \\
& >0\} d x=\int_{0}^{\infty} \int_{0}^{x} d\left(h^{\prime}(a)\right) \\
& \cdot P\left\{\left[\left(X_{\alpha}^{*}-x\right) \vee\left(X_{\alpha}^{* *}-x\right)\right]>0\right\} d x \\
& =\int_{0}^{\infty}\left(h^{\prime}(a)\right)_{0}^{x} P\left\{\left[\left(X_{\alpha}^{*}-x\right) \vee\left(X_{\alpha}^{* *}-x\right)\right]\right. \\
& >0\} d x=\int_{0}^{\infty}\left[h^{\prime}(x)-h^{\prime}(0)\right] \\
& \cdot P\left\{\left[\left(X_{\alpha}^{*}-x\right) \vee\left(X_{\alpha}^{* *}-x\right)\right]>0\right\} d x \\
& =\int_{0}^{\infty} h^{\prime}(x) P\left\{\left[\left(X_{\alpha}^{*}-x\right) \vee\left(X_{\alpha}^{* *}-x\right)\right]>0\right\} d x \\
& -h^{\prime}(0) \int_{0}^{\infty} P\left\{\left[\left(X_{\alpha}^{*}-x\right) \vee\left(X_{\alpha}^{* *}-x\right)\right]>0\right\} d x \\
& =\int_{0}^{\infty} h^{\prime}(x) P\left\{\left[\left(X_{\alpha}^{*}-x\right) \vee\left(X_{\alpha}^{* *}-x\right)\right]>0\right\} d x \\
& -h^{\prime}(0) E(X)=\int_{0}^{\infty} h^{\prime}(x) P\left\{\left[\left(X_{\alpha}^{*} \vee X_{\alpha}^{* *}\right)\right]\right. \\
& >x\} d x-h^{\prime}(0) E(X)=\int_{0}^{\infty} h^{\prime}(x)(1 \\
& \left.-P\left\{\left[\left(X_{\alpha}^{*} \vee X_{\alpha}^{* *}\right)\right] \leq x\right\} d x\right)-h^{\prime}(0) E(X) \\
& =\int_{0}^{\infty} h^{\prime}(x) \int_{x}^{\infty} d P\left\{\left[X_{\alpha}^{*} \vee X_{\alpha}^{* *}\right] \leq y\right\}-h^{\prime}(0) \\
& \cdot E(X)=\int_{0}^{\infty} \int_{x}^{\infty} h^{\prime}(x) d x d P\left\{X_{\alpha}^{*} \vee X_{\alpha}^{* *}\right\} \leq y \\
& -h^{\prime}(0) E(X) \\
& =\int_{0}^{\infty} \int_{0}^{y} h^{\prime}(x) d x\left[d P\left\{X_{\alpha}^{*} \vee X_{\alpha}^{* *}\right\} \leq y\right]-h^{\prime}(0) \\
& \cdot E(X)=\int_{0}^{\infty} h(y)\left[d P\left\{X_{\alpha}^{*} \vee X_{\alpha}^{* *}\right\} \leq y\right]-h(0) \\
& -h^{\prime}(0) E(X)=E[h(X)]-h(0)-h^{\prime}(0) E(X) \text {. }
\end{aligned}
$$

that is,

$$
\begin{aligned}
& \int_{0}^{\infty} h^{\prime \prime}(a) \int_{a}^{\infty} \bar{G}(x) d x d a \\
& \quad=E[h(Y)]-h(0)-h^{\prime}(0) E(Y) .
\end{aligned}
$$

By (15) we have

$$
\begin{aligned}
& E[h(X)]-h(0) h^{\prime}(0) E(X) \\
& \quad \geq E[h(Y)]-h(0)-h^{\prime}(0) E(Y),
\end{aligned}
$$

that is, $E[h(X)]-h^{\prime}(0) E(X)$

$$
\geq E[h(Y)]-h^{\prime}(0) E(Y),
$$

that is, $E[h(X)]-E[h(Y)]$

$$
\geq h^{\prime}(0)(E(X)-E(Y)) \text {. }
$$

By stipulation,

$$
\begin{aligned}
& \int_{a}^{\infty} P\left\{\left[\left(X_{\alpha}^{*}-x\right) \vee\left(X_{\alpha}^{* *}-x\right)\right]>0\right\} d x \\
& \quad \geq \int_{a}^{\infty} P\left\{\left[\left(Y_{\alpha}^{*}-x\right) \vee\left(Y_{\alpha}^{* *}-x\right)\right]>0\right\} d x
\end{aligned}
$$

for all $a \geq 0$.

Taking $a=0$,

$$
\begin{aligned}
& \int_{0}^{\infty} P\left\{\left[\left(X_{\alpha}^{*}-x\right) \vee\left(X_{\alpha}^{* *}-x\right)\right]>0\right\} d x \\
& \quad \geq \int_{0}^{\infty} P\left\{\left[\left(Y_{\alpha}^{*}-x\right) \vee\left(Y_{\alpha}^{* *}-x\right)\right]>0\right\} d x
\end{aligned}
$$

$$
E(X) \geq E(Y) .
$$

Since

$$
\begin{aligned}
& h^{\prime}(0) \geq 0, \\
& E(X) \geq E(Y) .
\end{aligned}
$$

We have

$$
E[h(X)]-E[h(Y)] \geq 0
$$

that is,

$$
E[h(X)] \geq E[h(Y)]
$$

which concludes the proof.

Theorem 15. If $X$ and $Y$ are nonnegative fuzzy random variables such that $E[X]=E[Y]$ then $X \geq_{v} Y$ if and only if $E[h(X)] \geq E[h(Y)]$ for all convex $h$.

Proof. Let $h$ be convex and suppose that $X \geq_{v} Y$.

By stipulation $E[X]=E[Y]$; then by (25) we have $E[h(X)] \geq E[h(Y)]$. 
Theorem 16. For the fuzzy random variables $X$ and $Y$ the following conditions are equivalent:

(i) $X<^{(P)} Y$.

(ii) $E\left(X^{p}-t^{p}\right)^{+} \leq E\left(Y^{p}-t^{p}\right)^{+}$.

(iii) $E\left(\Psi\left(X^{p}\right)\right) \leq E\left(\Psi\left(Y^{p}\right)\right)$ for all nondecreasing convex $\Psi$ on $[0, \infty)$.

Proof. Consider

$$
\begin{aligned}
& P^{-1} \int_{0}^{\infty}\left(x^{p}-t^{p}\right)^{+} d p\left\{\left(X_{\alpha}^{*}-x\right) \vee\left(X_{\alpha}^{* *}-x\right)\right\} \leq 0 \\
& =P^{-1} \int_{0}^{t}\left(x^{p}-t^{p}\right)^{+} d p\left\{\left(X_{\alpha}^{*}-x\right) \vee\left(X_{\alpha}^{* *}-x\right)\right\} \\
& \leq 0+P^{-1} \int_{t}^{\infty}\left(x^{p}-t^{p}\right)^{+} d p\left\{\left(X_{\alpha}^{*}-x\right) \vee\left(X_{\alpha}^{* *}-x\right)\right\} \leq 0 \\
& =P^{-1} \int_{t}^{\infty}\left(x^{p}-t^{p}\right) d p\left\{\left(X_{\alpha}^{*}-x\right) \vee\left(X_{\alpha}^{* *}-x\right)\right\} \leq 0 \\
& =\left.P^{-1} \int_{t}^{\infty}\left(x^{p}-t^{p}\right) p\left\{\left(X_{\alpha}^{*}-x\right) \vee\left(X_{\alpha}^{* *}-x\right)\right\}\right|_{t} ^{\infty} \\
& -P \int_{t}^{\infty} p\left\{\left(X_{\alpha}^{*}-x\right) \vee\left(X_{\alpha}^{* *}-x\right) \leq a\right\} P x^{P-1} d x \\
& =P^{-1} \int_{t}^{\infty} P x^{P-1} d x \\
& -P \int_{t}^{\infty} p\left\{\left(X_{\alpha}^{*}-x\right) \vee\left(X_{\alpha}^{* *}-x\right) \leq 0\right\} x^{P-1} d x \\
& =\int_{t}^{\infty} x^{P-1}\left(P\left\{\left(X_{\alpha}^{*}-x\right) \vee\left(X_{\alpha}^{* *}-x\right)>0\right\}\right) d x, \\
& X<{ }^{(P)} Y \Longleftrightarrow \int_{0}^{\infty} x^{P-1} P\left\{\left(X_{\alpha}^{*}-x\right) \vee\left(X_{\alpha}^{* *}-x\right)>0\right\} d x \\
& \leq \int_{0}^{\infty} x^{P-1} P\left\{\left(Y_{\alpha}^{*}-x\right) \vee\left(Y_{\alpha}^{* *}-x\right)>0\right\} d x ; \quad t \geq 0 \\
& \Longleftrightarrow P^{-1} \int_{t}^{\infty}\left(\left(x^{p}-t^{p}\right)^{+} d p\left\{\left(X_{\alpha}^{*}-x\right) \vee\left(X_{\alpha}^{* *}-x\right)\right\} \leq 0\right) \\
& \leq P^{-1} \int_{t}^{\infty}\left(y^{p}-t^{p}\right)^{+} d p\left(\left(Y_{\alpha}^{*}-x\right) \vee\left(Y_{\alpha}^{* *}-x\right) \leq 0\right) \\
& \Longleftrightarrow E\left(X^{p}-t^{p}\right)^{+} \leq E\left(Y^{p}-t^{p}\right)^{+} \text {. }
\end{aligned}
$$

Since $\Psi$ is nondecreasing convex function on $[0, \infty)$,

$$
\begin{aligned}
E\left(X^{p}-t^{p}\right)^{+} & \leq E\left(Y^{p}-t^{p}\right)^{+} \Longleftrightarrow E\left(\Psi\left(X^{p}\right)\right) \\
& \leq E\left(\Psi\left(Y^{p}\right)\right) .
\end{aligned}
$$

\section{Conclusion}

The variability ordering of fuzzy random variables is defined and as an application a property of new better than used in expectation and new worse than used in expectation is derived using variability orderings for fuzzy random variables. The generalized variability ordering and its weaker versions among nonnegative fuzzy random variables representing lifetime components are discussed. These orderings are employed to compare the appealing nature of different risks and are essential in the theory of decision making under uncertainty.

\section{Conflict of Interests}

The authors declare that there is no conflict of interests regarding the publication of this paper.

\section{References}

[1] H. Kwakernaak, "Fuzzy random variables-I. definitions and theorems," Information Sciences, vol. 15, pp. 1-29, 1978.

[2] H. Kwakernaak, "Fuzzy random variables-II," Information Sciences, vol. 17, pp. 153-178, 1979.

[3] M. L. Puri and D. A. Ralescu, "Fuzzy random variables," Journal of Mathematical Analysis and Applications, vol. 114, no. 2, pp. 409-422, 1986.

[4] M. C. Bhattacharjee, "Some generalized variability orderings among life distributions with reliability applications," Journal of Applied Probability, vol. 28, no. 2, pp. 374-383, 1991.

[5] A. M. Abouammoh, "On the criteria of the mean remaining life," Statistics \& Probability Letters, vol. 6, no. 4, pp. 205-211, 1988.

[6] M. C. Bhattacharjee and J. Sethuraman, "Families of life distributions characterized by two moments," Journal of Applied Probability, vol. 27, no. 3, pp. 720-725, 1990.

[7] E. J. L. Piriyakumar and N. Renganathan, "Stochastic orderings of fuzzy random variables," International Journal of Information Science and Management, vol. 12, no. 4, pp. 29-40, 2001.

[8] S. Ramasubramanian and P. Mahendran, "Estimation of hazard rate and mean residual life ordering for fuzzy random variable," Abstract and Applied Analysis, vol. 2015, Article ID 164795, 5 pages, 2015.

[9] S. M. Ross and Z. Schechner, "Some reliability applications of the variability ordering," Research Report ORC 82-6, University of California, 1982.

[10] R. E. Barlow and F. Proschan, Statistical Theory of Reliability and Life Testing, Probability Models, Holt, Rinehart and Winston, New York, NY, USA, 1975.

[11] E. Lorenzo, G. Malla, and H. Mukerjee, "Estimation of distributions with the new better than used in expectation property," Statistics \& Probability Letters, vol. 83, no. 5, pp. 1346-1352, 2013.

[12] L. A. Zadeh, "Fuzzy sets," Information and Computation, vol. 8, pp. 338-353, 1965.

[13] S. B. Boswell and M. S. Taylor, "A central limit theorem for fuzzy random variables," Fuzzy Sets and Systems, vol. 24, no. 3, pp. 331344, 1987.

[14] E. J. Lazarus Piriyakumar and S. Ramasubramanian, "Variability orderings of fuzzy random variables," in Proceedings of the National Conference on Discrete Mathematics and Its Applications (NCDMA '07), pp. 221-229, September 2007.

[15] M. Rausand and A. Hoyla, System Reliability Theory, Models, Statistical Methods and Applications, John Wiley \& Sons, 2004. 


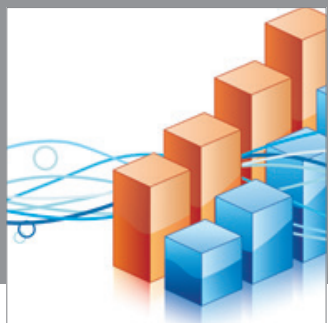

Advances in

Operations Research

mansans

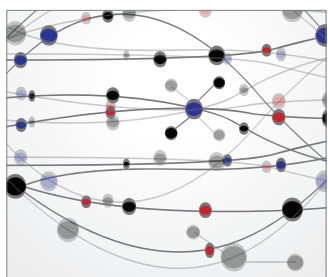

The Scientific World Journal
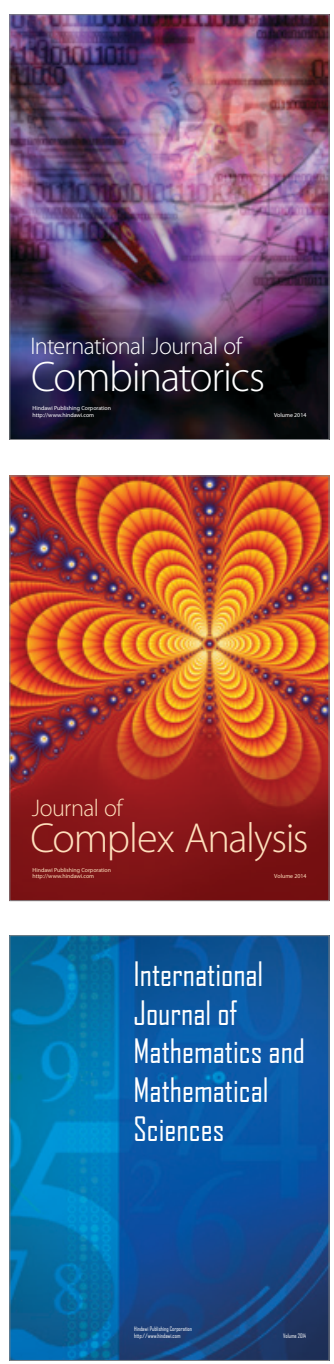
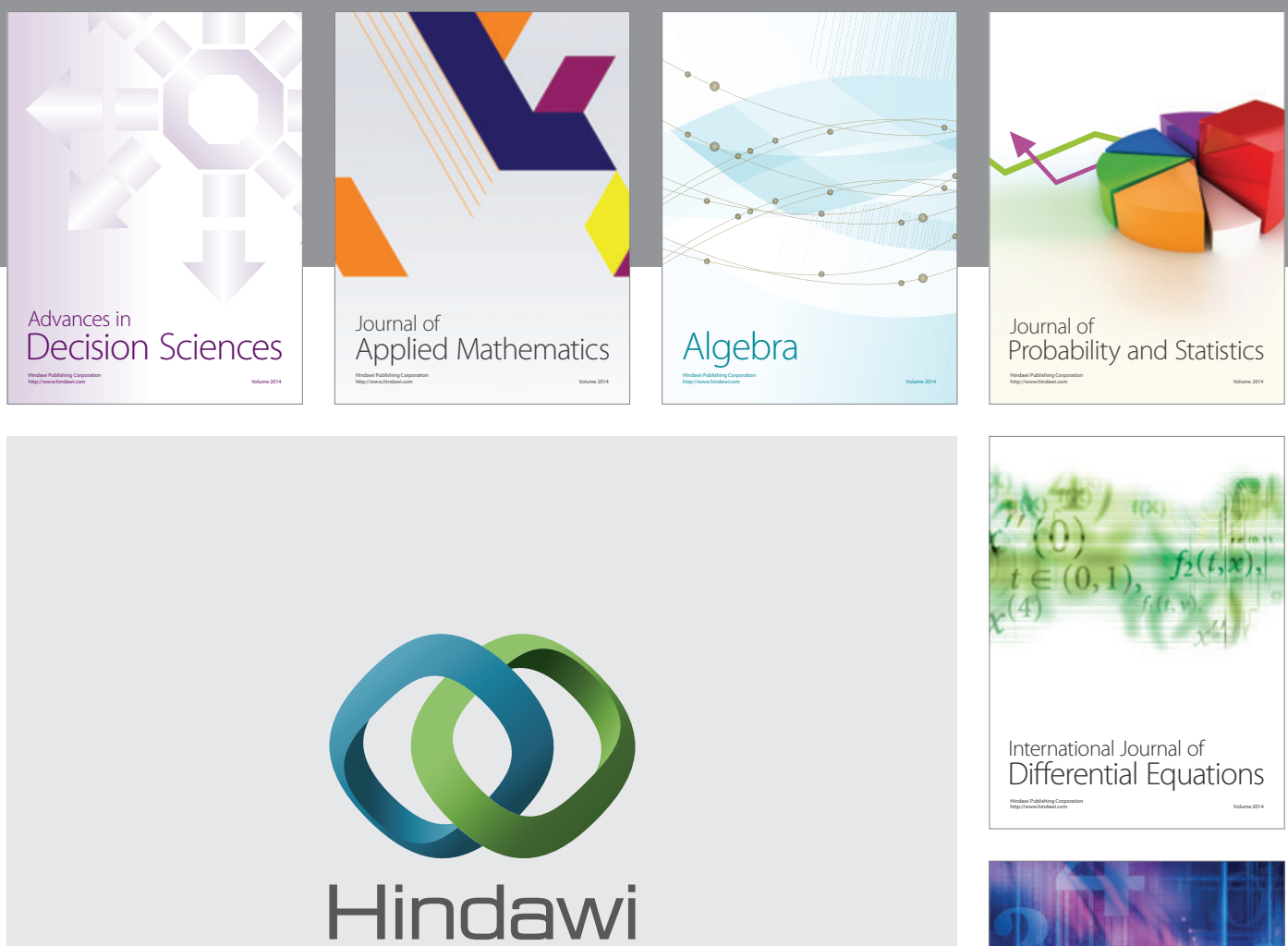

Submit your manuscripts at http://www.hindawi.com
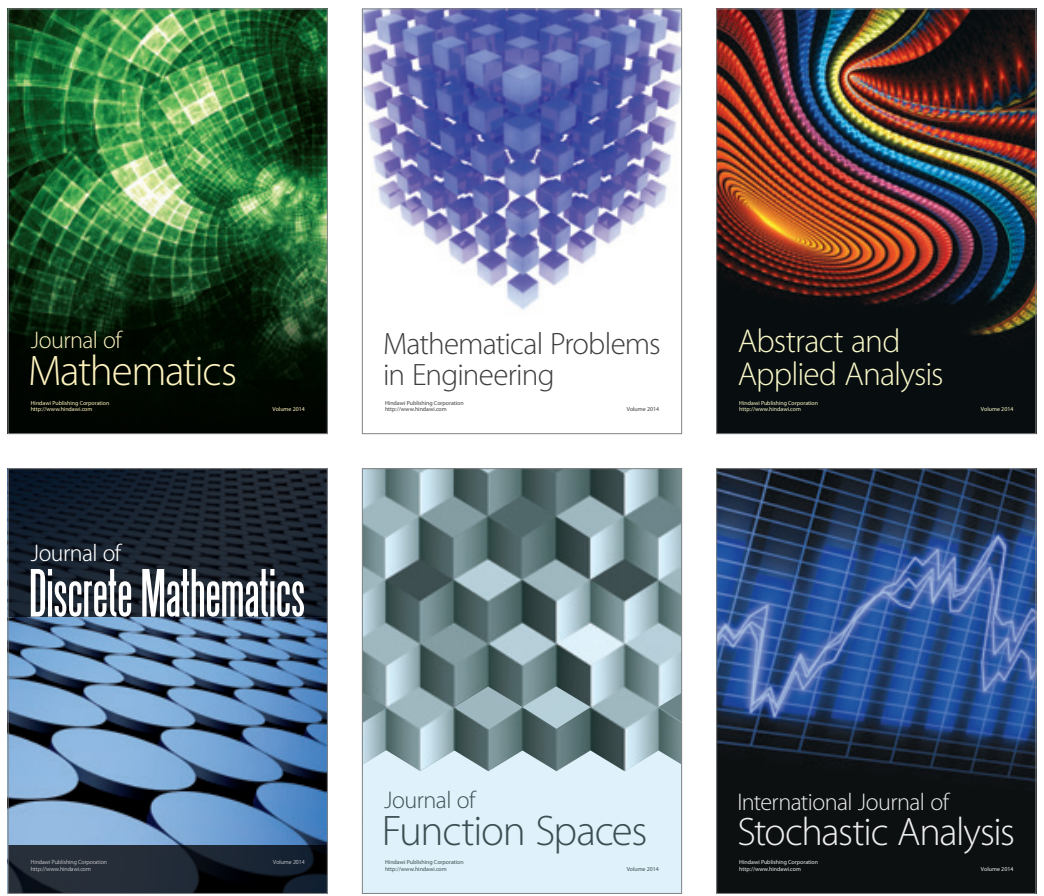

Journal of

Function Spaces

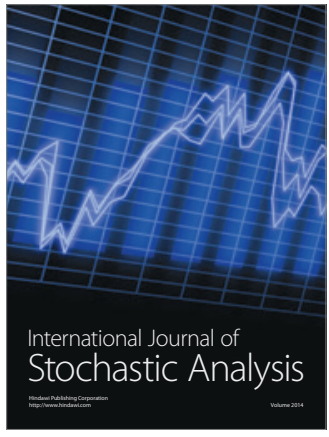

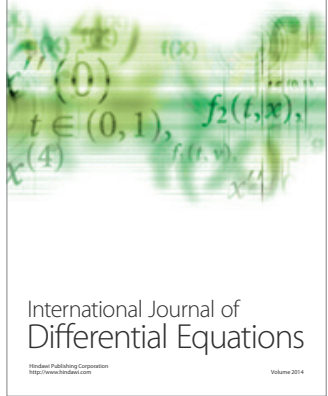
